\title{
Minichromosome maintenance protein 5 is an important pathogenic factor of oral squamous cell carcinoma
}

\author{
MIAO HAO ${ }^{1 *}$, HUIYU WANG $^{2 *}, \mathrm{CHU}^{2} \mathrm{ZHANG}^{2},{\mathrm{CHUNYAN} \mathrm{LI}^{2} \text { and XIAOFENG WANG }}^{2}$ \\ ${ }^{1}$ Scientific Research Center; ${ }^{2}$ Department of Stomatology, \\ China-Japan Union Hospital of Jilin University, Changchun, Jilin 130033, P.R. China
}

Received January 18, 2020; Accepted July 8, 2020

DOI: $10.3892 / \mathrm{ol} .2020 .11970$

\begin{abstract}
Oral squamous cell carcinoma is one of the most common causes of malignancy-associated death. Early diagnosis of oral squamous cell carcinoma (OSCC) is important in patient treatment and prognostic evaluation. Due to the lack of significant therapeutic benefit, the 5-year survival rate has not improved. Therefore, effective novel markers are needed to improve diagnosis. To determine novel promising diagnostic biomarkers for OSCC, 416 upregulated and 416 downregulated differentially expressed genes were screened from OSCC tissues using an RNA microarray. The results suggested that minichromosome maintenance protein (MCM5) mRNA was significantly overexpressed in OSCC tissues compared with that in adjacent normal tissues. Moreover, silencing of MCM5 expression an OSCC cell line (SCC-15) significantly impaired proliferation and colony formation. Furthermore, negative regulation of the mRNA and protein expression of MCM5 and demonstrated that MCM5 served as a cancer-promoting gene modulating OSCC cell proliferation through induced $\mathrm{G}_{2} / \mathrm{M}$ phase arrest. In this process, the mRNA expression of cyclin $\mathrm{E}$ and cyclin-dependent kinase 2 was downregulated, while p21 expression was upregulated. These results suggested that MCM5 may be an important pathogenic factor of OSCC. High expression levels of MCM5 may serve as a marker for the early diagnosis of OSCC.
\end{abstract}

\section{Introduction}

Oral squamous cell carcinoma (OSCC) affects $>600,000$ individuals worldwide annually (1). Presently, the clinical treatment of OSCC is primarily surgery, radiotherapy or chemotherapy $(2,3)$. Over the past decades, the overall survival

Correspondence to: Dr Xiaofeng Wang, Department of Stomatology, China-Japan Union Hospital of Jilin University, 126 Xiantai, Changchun, Jilin 130033, P.R. China

E-mail: wangxiaofeng@jlu.edu.cn

${ }^{*}$ Contributed equally

Key words: minichromosome maintenance protein 5, cell cycle, oral squamous cell carcinoma, biomarker, microarray
(OS) rate of patients with OSCC has not significantly improved, with a 5-year survival rate of $29-45 \%$ (4). Insufficient sensitive and specific biomarkers may lead to the diagnosis of OSCC at advanced stages (5). Therefore, it is necessary to identify novel biomarkers for the early diagnosis and treatment of OSCC.

Recently, with the continuous development of sequencing technology, researchers can efficiently distinguish differentially expressed genes (DEGs) by transcriptome sequencing, which allows screening of potential tumor markers or therapeutic drug targets (6). For example, a number of new potential tumor markers have been found in human malignancies, such as breast cancer (7), epithelial ovarian cancer (8) and glioma (9).

Minichromosome maintenance protein 5 (MCM5), a member of mini-chromosome maintenance family of proteins, plays an important role in cell proliferation and DNA replication $(10,11)$. Some studies have confirmed that MCM5 is highly expressed in numerous human malignancies, such as renal cell carcinoma (12), pancreatic ductal adenocarcinoma (13), cervical cancer (14) and skin cancer (15). Further studies have found that high expression of MCM5 is closely associated with the clinicopathological features of specific cancer types. For example, overexpression of MCM5 is significantly associated with overall survival rate (OS) in hepatocellular carcinoma (16). Moreover, increased expression of MCM5 is positively correlated with larger tumor size, positive lymph node metastasis, more advanced clinical stage, higher histological grade, deeper invasion depth and perineural invasion of OSCC (17). However, thus far, the expression, function and potential mechanisms of MCM5 in OSCC are still unclear. Therefore, the present study aimed to analyze the DEGs in OSCC using a microarray, screen for MCM5 and further evaluate the possible functions of MCM5 in OSCC. The present results may provide evidence to support the value of MCM5 as a biomarker or a therapeutic target of OSCC.

\section{Materials and methods}

Tissue sampling. Pairs of OSCC tissues and adjacent normal tissues were obtained from 18 patients undergoing resection operations at the China-Japan Union Hospital of Jilin University (Changchun, China). Clinicopathological data were also collected. No patient received preoperative treatment, including radiotherapy or chemotherapy. No other inclusion/exclusion criteria were used. Matched normal OSCC 
tissues were obtained from a segment of the resected specimens $>5-\mathrm{cm}$ away from the tumor. Pathological analysis was used to identify surgically resected specimens. Pathological analysis was performed by our group with no specific diagnostic guidelines. Three paired samples were obtained for transcriptome sequencing. Then, to confirm the reliability of sequencing data, the samples size was increased using the remaining 15 paired tissues and analyzed using quantitative (q)PCR. All comparisons between OSCC tissues and adjacent normal tissues were performed simultaneously. The Kaplan-Meier analysis of OS and survival curves were from the Cancer Genome Atlas database (TCGA, https://www.cancer.gov/).

The study was approved by the Ethics Committee of China-Japan Union Hospital of Jilin University. Written informed consent was obtained from all patients who participated in this study.

Transcriptome sequencing and functional annotation analysis. Total RNA extraction, RNA library construction and transcriptome sequencing were performed at Sangon Biotech Co., Ltd. The biological relevance of unique genes in expression profiles of DEGs were screened according to the threshold values of $\log _{2}$ Ifold-changel $\geq 1$ and $\mathrm{P}<0.05$. Then the differentially expressed mRNAs were analyzed by Gene Ontology (GO) whose annotations were downloaded from Gene Ontology (http://geneontology.org/), UniProt (https://sparql.uniprot.org/) and NCBI (https://www.ncbi.nlm. nih.gov/). Significant GO categories were identified using Fisher's exact test with a $\mathrm{P}<0.05$, which indicated that significantly upregulated genes in the set of DEGs were assigned to a specific functional category more often than expected by chance. Significant pathways of the DEGs were then analyzed and identified according to the Kyoto Encyclopedia of Genes and Genomes (KEGG) database (https://www.kegg.jp/).

Cell lines. The human tongue squamous cell carcinoma SCC-15 and CAL-27 were obtained from the American Type Culture Collection. CAL-27 cells were cultured in DMEM medium with $10 \%$ fetal bovine serum (FBS) (Gibco; Thermo Fisher Scientific, Inc.), $100 \mathrm{U} / \mathrm{ml}$ penicillin and streptomycin at $37^{\circ} \mathrm{C}$ in a humidified atmosphere containing $5 \% \mathrm{CO}_{2}$. SCC-15 cells were cultured in MEM medium with $10 \% \mathrm{FBS}, 1 \%$ NEAA, $100 \mathrm{U} / \mathrm{ml}$ penicillin and streptomycin at $37^{\circ} \mathrm{C}$ in a humidified atmosphere containing $5 \% \mathrm{CO}_{2}$.

MCM5-specific siRNA and transfection. Three MCM5 siRNA sequences were synthesized by Suzhou GenePharma Co., Ltd. The sequences were as follows (5'-3'): siRNA-1, Forward: CCGACUACUUGUACAAGCATT and reverse: UGCUUG UACAAGUAGUCGGTT; siRNA-2, forward: CCAAAUGUC UAUGAGGUCATT and reverse: UGACCUCAUAGACAU UUGGTT, siRNA-3, forward: GUCGUCUGUAUUGACGAG UTT and reverse: ACUCGUCAAUACAGACGACTT and scrambled, forward: UUCUCCGAACGUGUCACGUTT and reverse: ACGUGACACGUUCGGAGAATT. The mock was an untransfected empty vector, serving as the control.

SCC-15 cells $\left(4.5 \times 10^{4}\right.$ cells/well $)$ were cultured in 6 -well plates overnight at $37^{\circ} \mathrm{C}$. Then, cells were transfected with $50 \mathrm{nM}$ negative control siRNA or MCM5 siRNA using Lipofectamine ${ }^{\circledR} 2000$ Transfection Reagent (Invitrogen;
Thermo Fisher Scientific, Inc.) according to the manufacturer's protocol. After $48 \mathrm{~h}$ transfection, cells were collected, and then RNA was extracted by TRIzol ${ }^{\circledR}$ regent (Invitrogen; Thermo Fisher Scientific, Inc.) for further experiments as indicated.

Reverse transcription (RT) qPCR. RNA extracted from tissues samples were reverse transcribed into cDNA using a GoScript Reverse Transcription System kit (Monad; http://www.monadbiotech.com/) according to the manufacturer's instructions. Relative mRNA expressions were quantified by qPCR using the QuantiTect SYBR Green PCR kit (Roche Diagnostics) and normalized to GAPDH using primers listed in Table I. The cycling parameters were 40 cycles of $95^{\circ} \mathrm{C}$ for $15 \mathrm{sec}, 60^{\circ} \mathrm{C}$ for $15 \mathrm{sec}$ and $72^{\circ} \mathrm{C}$ for $30 \mathrm{sec}$. Relative mRNA levels were assessed by the comparative $2^{-\Delta \Delta \mathrm{Cq}}$ method (18). All analyses of the samples were conducted in triplicate.

For association of MCM5 expression levels with clinicopathologic features of OSCC, the relative expression levels of MCM5 were evaluated using qPCR as aforementioned. Relative mRNA levels of paired samples (adjacent vs. cancer tissues) were assessed by the comparative $2^{-\Delta \Delta \mathrm{Cq}}$ method. A ratio $>1$ was considered to have high MCM5 expression, whereas a ratio $\leq 1$ was considered to have low MCM5 expression.

Cell proliferation assay. To analyze cell proliferation, a Cell Counting Kit (CCK)-8 (Dojindo Molecular Technologies, Inc.) was used according to the manufacturer's instructions. In total, 5,000 cells were cultured to each well of 96-well plates. After $24 \mathrm{~h}$ post siRNA transfection, cells were incubated for 24,48 and $72 \mathrm{~h}$. CCK-8 reagent was added $2 \mathrm{~h}$ prior to detection. The OD was measured at $450 \mathrm{~nm}$ using a microplate reader (Bio-Tek). The experiment was performed three times.

Colony formation assay. This assay was performed according to a previous study (19). Briefly, cells were cultured in 6-well plates at 1,000 cells/well, followed by culture in complete medium (DMEM supplemented with $10 \%$ FBS, $100 \mathrm{U} / \mathrm{ml}$ penicillin and streptomycin) for 2 weeks. The colonies were fixed with methanol for $15 \mathrm{~min}$ at room temperature and washed with PBS and stained with $0.1 \%$ crystal violet at room temperature (Beyotime Institute of Biotechnology) solution for $20 \mathrm{~min}$. A cell colony was defied as a group formation of at least 50 cells. Finally, formed colonies were observed and images were captured under a light microscope at magnification, x200.

Cell migration analysis using scratching assays. SCC-15 cells were cultured in a 6 -well plate at $5 \times 10^{5}$ cells/well overnight at $37^{\circ} \mathrm{C}$. Then the cells were scratched and scraped with fresh DMEM. Cells were observed and images were captured under a light microscope (magnification, $\mathrm{x} 200$ ) at $0 \mathrm{~h}$. The width of the scratch was measured and referred to as $\mathrm{W}_{\text {before }}$. Then, the cells were starved with no FBS and returned to the incubator for $6 \mathrm{~h}$ at $37^{\circ} \mathrm{C}$. The width of the same scratch was measured and referred to as $\mathrm{W}_{\mathrm{after}}$. Migrating distance was calculated by subtracting $\mathrm{W}_{\text {after }}$ from $\mathrm{W}_{\text {before }}$. The migration of the control was set as 100 .

Western blot analysis. The protein extractions from the cells were isolated using RIPA Lysis Buffer (P0013B; Beyotime Institute of Biotechnology). Then, 50-100 $\mu \mathrm{g}$ protein was loaded per lane on a $12 \%$ gel, resolved using SDS-PAGE and electroblotted onto 
Table I. Primers used for reverse transcription quantitative-PCR.

\begin{tabular}{lll}
\hline mRNA & \multicolumn{1}{c}{ Forward primer $\left(5^{\prime}-3^{\prime}\right)$} & \multicolumn{1}{c}{ Reverse primer $\left(5^{\prime}-3^{\prime}\right)$} \\
\hline$M C M 5$ & GATCCTGGCATTTTCTACAG & CCCTGTATTTGAAGGTGAAG \\
$P 21$ & GGAGACTCTCAGGGTCGAAA & GGATTAGGGCTTCCTCTTGG \\
CyclinE & TTCTTGAGCAACACCCTCTTCTGCAGCC & TCGCCATATACCGGTCAAAGAAATCTTGTGCC \\
$C D K 2$ & GCTAGCAGACTTTGGACTAGCCAG & AGCTCGGTACCACAGGGTCA \\
GAPDH & AGAAGGCTGGGGCTCATTTG & AGGGGCCATCCACAGTCTTC
\end{tabular}

MCM5, minichromosome maintenance protein; cyclin-dependent kinase 2.

A

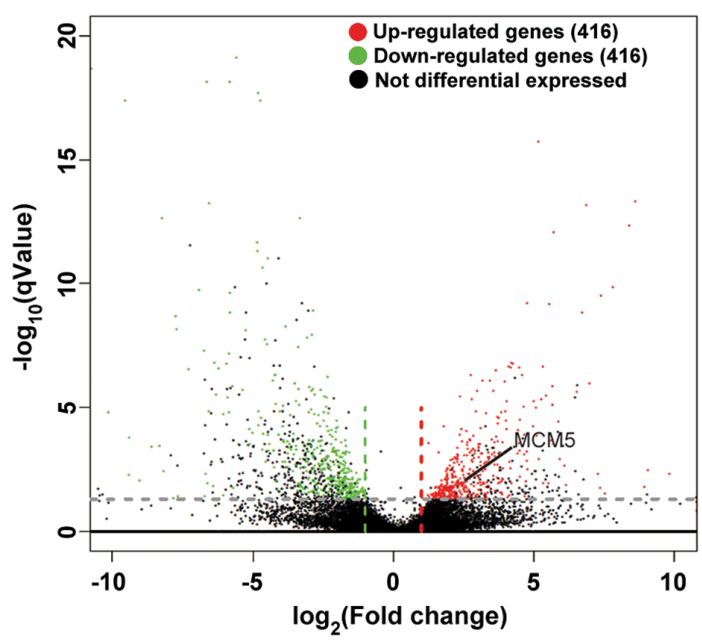

B

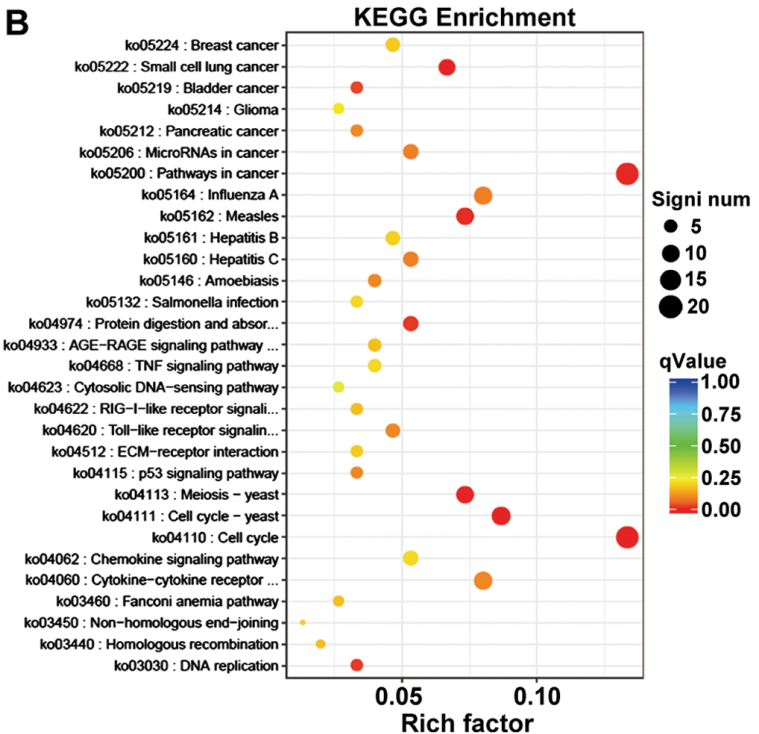

Figure 1. Volcano plots and KEGG pathway analysis of differentially expressed genes between OSCC cancer tissue group and adjacent normal tissue group. (A) Differences in gene expression profiles between OSCC cancer tissue group and adjacent normal tissue group. The horizontal line corresponds to a 2 -fold $\left(\log _{2}\right.$ scaled) change up or down, and the vertical line represents $\mathrm{P}<0.05$. The red points on the plot represent the differentially expressed genes with a 2 -fold change upregulation while the green points represent downregulation with $\mathrm{P}<0.05)$. (B) Top 30 KEGG enrichment terms of DEG in OSCC. The vertical axis represents the pathway category and the horizontal axis represents the enrichment score $[-\lg (\mathrm{P}$-value $)]$ of the pathway. $\mathrm{LgP}$ was the $\operatorname{logarithm}$ of $\mathrm{P}$-value, and $\mathrm{P}<0.05$ was considered significant. KEGG, Kyoto Encyclopedia of Genes and Genomes; OSCC, oral squamous cell carcinoma; DEG, different expressed genes; OSCC, oral squamous cell carcinoma.

PVDF membranes (Roche Diagnostics). After blocking at $37^{\circ} \mathrm{C}$ for $1 \mathrm{~h}(5 \%$ non-fat milk in PBS plus $0.1 \%$ Tween 20$)$, the blots were incubated with primary antibodies against anti-MCM5 (D220960-0025; 1:1,000; BBI Life Sciences), anti-p21 (0053657; 1:1,000; Proteintech), anti-cyclin E (0047453; 1:1,000; ProteinTech Group, Inc.) and anti- $\beta$-actin (D16H11; 1:1,000; CST Biological Reagents Co., Ltd.) at $37^{\circ} \mathrm{C}$ for $1 \mathrm{~h}$. Western blots were probed with secondary antibodies and detected using the Odyssey infrared imaging system (LI-COR Biosciences).

Cell cycle analysis. SCC-15 cells were harvested and fixed with $70 \%$ ethanol on ice for $30 \mathrm{~min}$, and then washed with PBS to decant the ethanol solution. Then the cells were suspended and stained by PI and RNase A treatment. Cell cycle analysis was performed using a flow cytometer (FACSARIAII; BD Biosciences). The data was performed using CXP Analysis software (Beckman Coulter, Inc).

Statistical analysis. All the data analysis was performed using SPSS version 18.0 (SPSS, Inc.). The results are presented as mean + SD. Associations between MCM5 mRNA expression and clinicopathological factors were analyzed using the Pearson's $\chi^{2}$ test or Fisher's exact test. The differences in MCM5 mRNA expression between carcinoma and adjacent normal tissues were evaluated by a paired t-test. One-way ANOVA followed by Tukey's post hoc test was used to determine the differences between groups, and unpaired t-tests for the rest of the data. The survival rate was calculated by the Kaplan-Meier method and compared using the log-rank test. $\mathrm{P}<0.05$ was considered to indicate a statistically significant difference. All experiments were performed in triplicate.

\section{Results}

RNA sequencing and functional annotation analysis. To explore novel biomarkers for OSCC, the RNAs derived from tissue samples by sequencing were detected. Three matched primary OSCC tissues and adjacent normal tissues were randomly selected. As shown in Fig. 1A, the aberrant expression of genes was detected in tissue samples. To screen 
Table II. Twenty randomly selected differentially expressed genes between oral squamous cell carcinoma and adjacent tissue samples. The genes selected randomly instead of listing based on rank or fold-change in expression.

\begin{tabular}{|c|c|c|c|c|}
\hline Gene ID & $\log _{2}$ fold-change & P-value & Result & Gene name \\
\hline ENSG00000160182 & -18.41080047 & $8.81 \times 10^{-6}$ & Downregulation & $T F F 1$ \\
\hline ENSG00000205592 & -15.71681384 & $4.68 \times 10^{-5}$ & Downregulation & MUC19 \\
\hline ENSG00000171195 & -9.405322765 & $1.44 \times 10^{-6}$ & Downregulation & $M U C 7$ \\
\hline ENSG00000126549 & -8.237011727 & $1.40 \times 10^{-16}$ & Downregulation & STATH \\
\hline ENSG00000090382 & -6.918550063 & $2.09 \times 10^{-13}$ & Downregulation & $L Y Z$ \\
\hline ENSG00000161798 & -6.421368217 & $5.28 \times 10^{-4}$ & Downregulation & $A Q P 5$ \\
\hline ENSG00000161055 & -5.852302695 & $9.59 \times 10^{-8}$ & Downregulation & $S C G B 3 A 1$ \\
\hline ENSG00000107562 & -4.504588372 & $6.04 \times 10^{-11}$ & Downregulation & CXCL12 \\
\hline ENSG00000214711 & -3.313192374 & $6.01 \times 10^{-4}$ & Downregulation & CAPN14 \\
\hline ENSG00000106066 & -2.169225527 & $8.46 \times 10^{-6}$ & Downregulation & $C P V L$ \\
\hline ENSG00000184330 & 16.33797846 & $2.34 \times 10^{-14}$ & Upregulation & $S 100 A 7 A$ \\
\hline ENSG00000137745 & 9.059136388 & $7.53 \times 10^{-5}$ & Upregulation & $M M P 13$ \\
\hline ENSG00000243207 & 8.610243304 & $2.22 \times 10^{-17}$ & Upregulation & $P P A N-P 2 R Y 11$ \\
\hline ENSG00000107159 & 7.805639054 & $1.61 \times 10^{-13}$ & Upregulation & CA9 \\
\hline ENSG00000183072 & 6.976257283 & $3.80 \times 10^{-9}$ & Upregulation & $N K X 2-5$ \\
\hline ENSG00000196611 & 5.435774905 & $1.54 \times 10^{-8}$ & Upregulation & $M M P 1$ \\
\hline ENSG00000171217 & 4.773764562 & $7.05 \times 10^{-5}$ & Upregulation & CLDN20 \\
\hline ENSG00000178445 & 4.489639485 & $7.51 \times 10^{-6}$ & Upregulation & $G L D C$ \\
\hline ENSG00000100297 & 2.043710595 & $5.65 \times 10^{-4}$ & Upregulation & MCM5 \\
\hline ENSG00000127564 & 2.036248873 & $2.40 \times 10^{-5}$ & Upregulation & PKMYT1 \\
\hline
\end{tabular}

the candidate biomarkers for diagnosing OSCC, the DEGs were selected when changes in RNA expression were $>2$ fold-changes. As shown in Fig. 1A, 416 aberrant RNAs were significantly downregulated, while 416 RNAs were upregulated. In order to represent all differentially expressed genes, twenty randomly selected dysregulated genes between OSCC and adjacent tissue samples are summarized in Table II. The P-value and $\log _{2}$ fold-changes of all aberrant expression genes are shown in Table SI. The DEGs were selected randomly instead of listing based on rank or fold-change in expression.

To explore the role of differentially expressed RNAs in OSCC, KEGG pathway analysis was performed. Depending on the P-value and enrichment, 261 signal pathways associated with OSCC were identified (Table SII). The top 30 KEGG enrichment terms of DEGs are shown in Fig. 1B, including 'cell cycle', 'pathways in cancer', 'cell cycle-yeast', 'meiosis-yeast' and 'cytokine-cytokine receptor interaction'. Among these, it was reported that some genes, such as MCM5, cell division cycle 7-related protein kinase and Cyclin-dependent kinase 4 homolog, were primarily enriched in the 'cell cycle' pathway. Some genes, such as lysozyme C, statherin and aquaporin-5, were enriched in the "saliva secretion' pathway. MCM5, which participated in cell cycle regulation and had high expression in OSCC, was selected for further study and it was hypothesized that MCM5 might be a candidate tumor marker for OSCC.

Validation of MCM5 using RT-qPCR. To further verify the aforementioned expression profile data, MCM5 expression levels were investigated using RT-qPCR in 15 tumor and

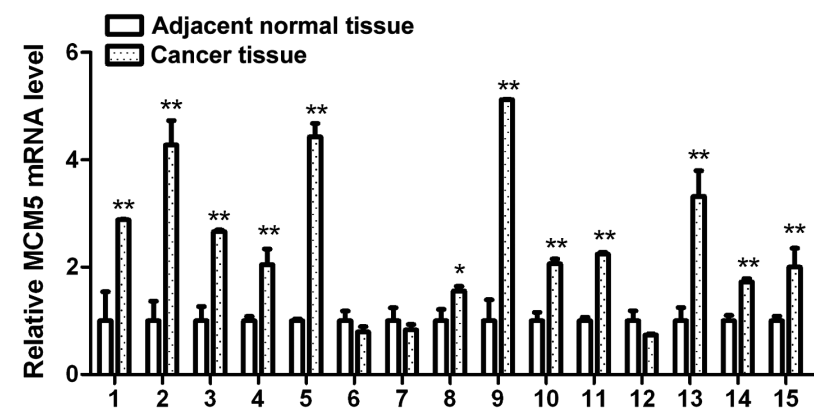

Figure 2. Relative expression levels of MCM5 mRNA in 15 paired adjacent normal tissues and oral squamous cell carcinoma tissues using quantitative PCR. The relative expression data were analyzed by the $2^{-\Delta \Delta C q}$ method. GAPDH was used as an internal control. ${ }^{*} \mathrm{P}<0.05,{ }^{* *} \mathrm{P}<0.01$ vs. adjacent normal tissue. MCM5, minichromosome maintenance protein.

adjacent normal tissues. As shown in Fig. 2, MCM5 mRNA was significantly upregulated in $80.0 \%$ (12/15) of tumor tissues compared with that in matched normal tissues. These results showed that MCM5 was highly expressed in OSCC tissues, which was in line with the sequencing data.

Association of MCM5 expression levels with clinicopathologic features of OSCC and survival analysis. The results of the potential association between MCM5 expression and clinicopathological features in 15 patients with OSCC are presented in Table III. No significant association with MCM5 expression was found for age, sex, histological differentiation, metastasis/recurrence and survival status $(\mathrm{P}>0.5)$. 
Table III. Association between expression of MCM5 and clinicopathologic features of 15 patients with oral squamous cell carcinoma.

\begin{tabular}{|c|c|c|c|c|}
\hline \multirow[b]{2}{*}{ Characteristic } & \multirow[b]{2}{*}{ Value, $\mathrm{n}$} & \multicolumn{2}{|c|}{ MCM5 expression } & \multirow[b]{2}{*}{ P-value } \\
\hline & & High, $n=12$ & Low, $n=3$ & \\
\hline Age, years & & & & 0.792 \\
\hline$<60$ & 6 & 5 & 1 & \\
\hline$\geq 60$ & 9 & 7 & 2 & \\
\hline Sex & & & & 0.519 \\
\hline Male & 12 & 10 & 2 & \\
\hline Female & 3 & 2 & 1 & \\
\hline Histological differentiation & & & & 0.438 \\
\hline Well and Moderate & 8 & 7 & 1 & \\
\hline Poor & 7 & 5 & 2 & \\
\hline Metastasis/Recurrence & & & & 0.605 \\
\hline Yes & 8 & 6 & 2 & \\
\hline No & 7 & 6 & 1 & \\
\hline Survival status & & & & 0.438 \\
\hline Death & 7 & 5 & 2 & \\
\hline Survival & 8 & 7 & 1 & \\
\hline
\end{tabular}

A Kaplan-Meier analysis of OS is shown in Fig. 3. Analysis of clinical data from TCGA showed that high MCM5 expression was no associated with a shorter OS in patients with OSCC (log-rank $\mathrm{P}=0.62$ ). These results suggested that MCM5 might not be a prognostic biomarker for OSCC.

Inhibitory effect of MCM5 in OSCC cell lines. To determine the functional role of MCM5, first, MCM5 expression was analyzed using RT-qPCR in two OSCC cell lines. Notably, SCC-15 cells expressed significantly higher levels of MCM5 compared with Cal-27 cells ( $\mathrm{P}<0.01$; Fig. 4A). Considering that knockdown of MCM5 in the cell line with high MCM5 expression may bring about more significant changes, the SCC-15 cell line was selected for further investigation of the functional role of MCM5. Three specific siRNA sequences were designed to inhibit MCM5 expression and transfected in SCC-15 cells, and the impact on MCM5 expression was determined using RT-qPCR. As shown in Fig. 4B, siRNA1, siRNA2 and siRNA3 transfection decreased MCM5 expression by 65.1( $\mathrm{P}<0.01), 31.7$ $(\mathrm{P}<0.01)$ and $11.0(\mathrm{P}<0.01)$, respectively, compared with the negative control. Then, the efficiencies were confirmed using western blotting (Fig. 4C). The inhibitory effect of siRNA1 and siRNA2 was significant, but not found in siRNA3. The results were consistent with those of RNA expression. siRNA1 transfection reduced MCM5 expression significantly in SCC-15 cells. Therefore, siRNA1 was used for subsequent experiments.

Effect of MCM5 inhibition on proliferation, colony formation and migration. To determine whether MCM5 regulates cell cycle and modulates cell proliferation in OSCC, the effect of inhibiting MCM5 expression on SCC-15 cell proliferation was investigated. As shown in Fig. 5A, the results showed that downregulation of MCM5 had significant anti-proliferative

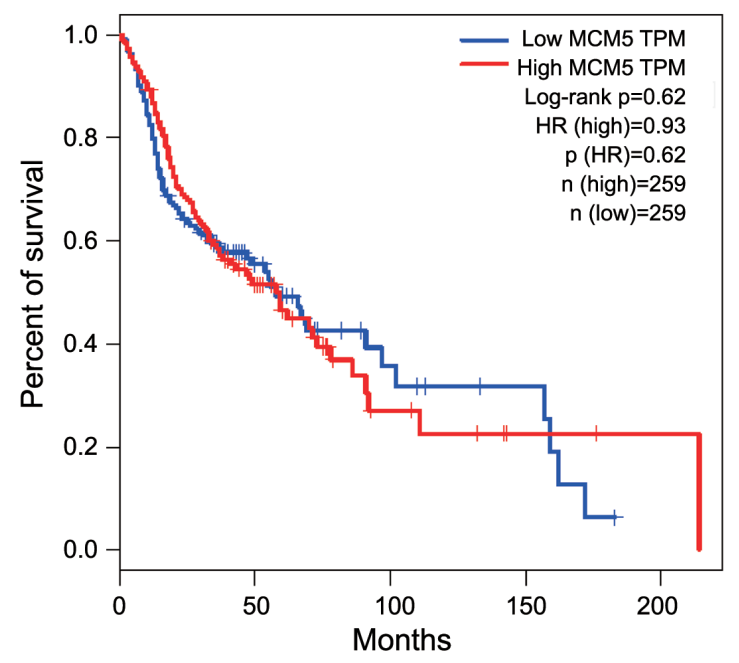

Figure 3. Survival curves from The Cancer Genome Atlas datasets $(n=518)$ containing high and low MCM5 expression levels. MCM5, minichromosome maintenance protein; HR, hazard ratio; TPM, transcripts per million.

effect compared with the negative control $(\mathrm{P}<0.01)$. Colony formation assays were performed, and the results revealed that, compared with the number of colonies in the control group, downregulation of MCM5 significantly reduced colony formation $(\mathrm{P}<0.01$; Fig. $5 \mathrm{~B}$ and $\mathrm{C})$. To estimate the impact of MCM5 on OSCC migration, scratching assays were conducted, and inhibition of MCM5 showed no significant impact on the migration of SCC-15 cells $(\mathrm{P}>0.05$; Fig. 5D). These results suggested that inhibiting MCM5 expression inhibited cell proliferation and colony formation, but had no effect on migration in SCC-15 cells. 
A

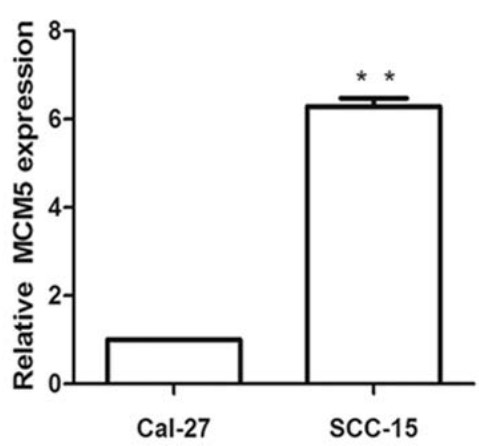

C
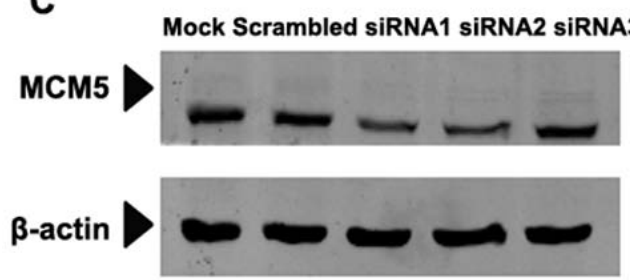

B

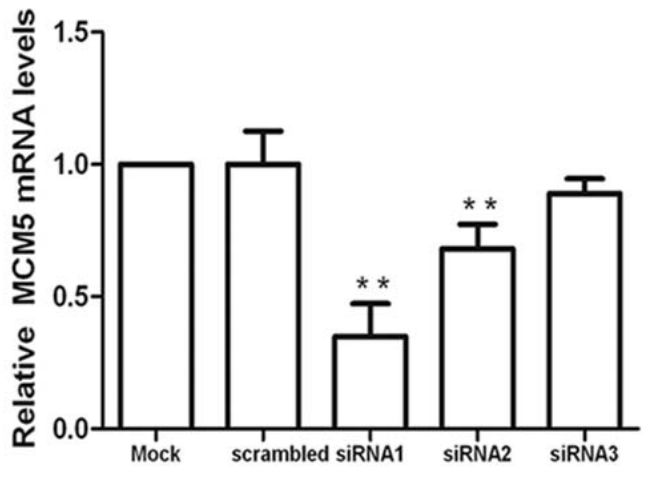

Figure 4. Inhibition of MCM5 expression in oral squamous cell lines. (A) MCM5 expression in Cal-27 and SCC-15 cells. (B) Efficiency of siRNA-MCM5 was confirmed by reverse transcription quantitative-PCR in SCC-15 cells. (C) The efficiency of siRNA-MCM5 was confirmed by western blot in SCC-15 cells. MCM5, minichromosome maintenance protein; si, small interfering; NC, negative control.

A

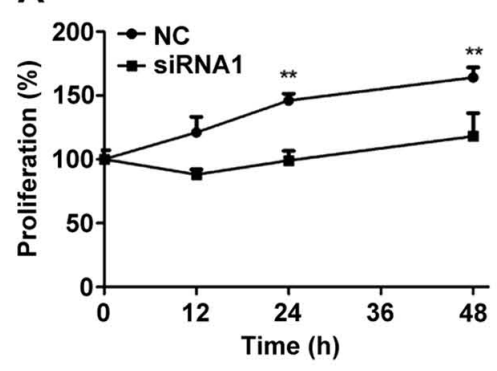

B
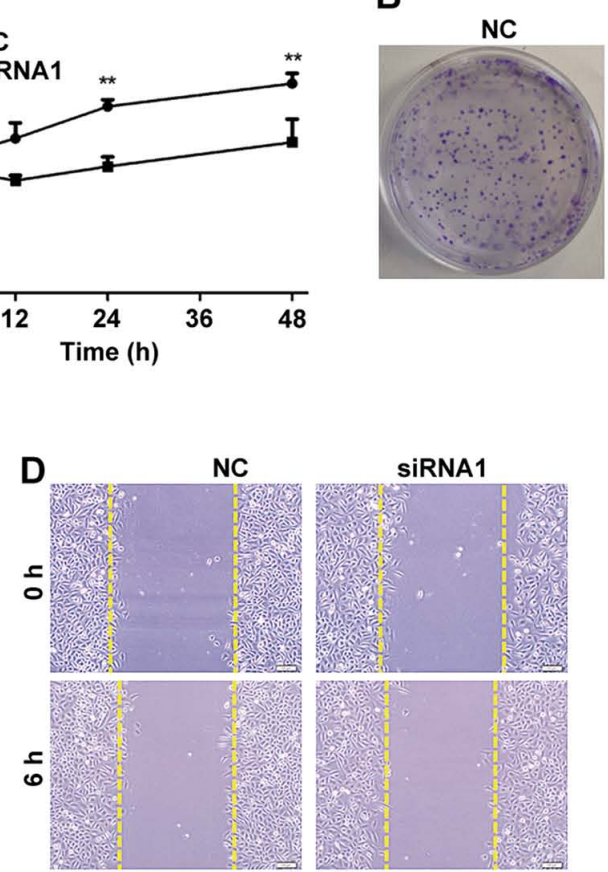

SiRNA1

NC

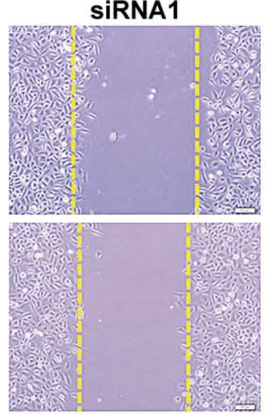

siRNA1

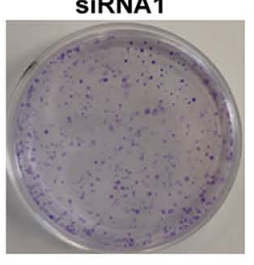

C

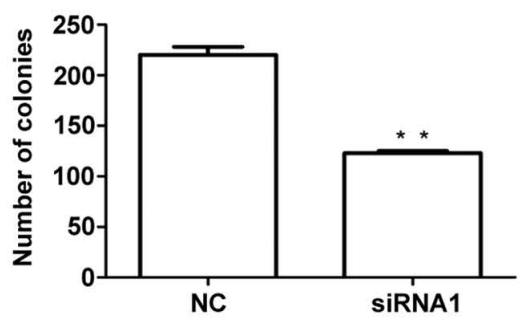

E

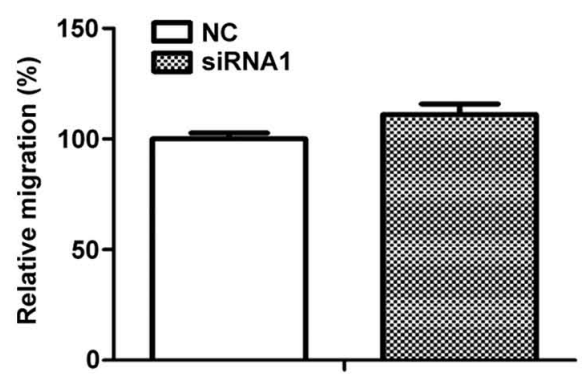

Figure 5. Effects of MCM5 inhibition on antiproliferation, colony formation and migration capacity in SCC-15 cells. (A) Downregulation of MCM5 expression suppressed SCC-15 cell proliferation compared with the corresponding control at different time points. (B) Downregulation of MCM5 expression inhibited SCC-15 cells colony formation compared with the corresponding control. (C) Quantification of MCM5 inhibition on colony formation compared with the corresponding control. (D) Cells scratching wounds observed by microscopy. (E) Downregulation of MCM5 had no effect on the migration capacity of SCC-15 cells. ${ }^{* *} \mathrm{P}<0.01$ vs. NC. MCM5, minichromosome maintenance protein; si, small interfering; NC, negative control.

Effect of MCM5 inhibition on cell cycle. To determine the potential mechanism for the observed proliferation inhibition of SCC-15 cells by MCM5 inhibition, cell cycle analysis was performed using flow cytometry. As shown in Fig. 6A and B, after MCM5 inhibition, the number of cells were decreased in the $G_{0} / G_{1}$ phase and the $S$ phase but significantly increased in the $\mathrm{G}_{2} / \mathrm{M}$ phase compared with the negative control. These results indicated that MCM5 inhibition significantly induced $\mathrm{G}_{2} / \mathrm{M}$ phase arrest compared with that in the control group. 
A

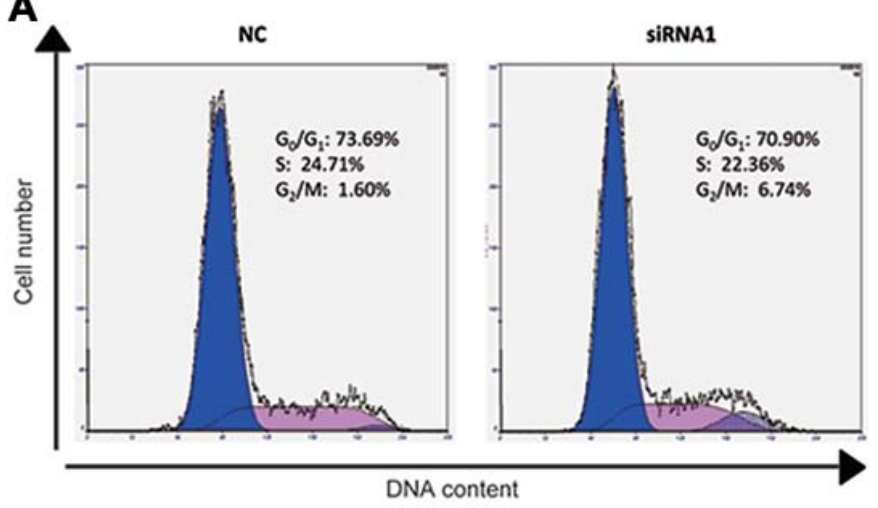

B

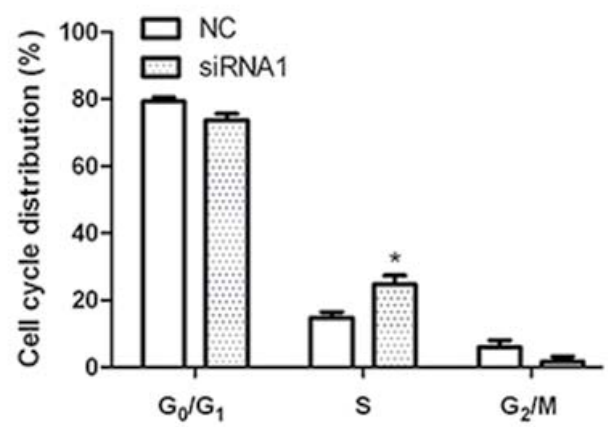

C

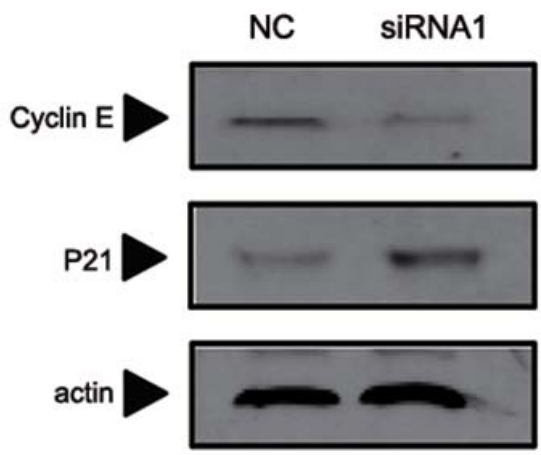

D

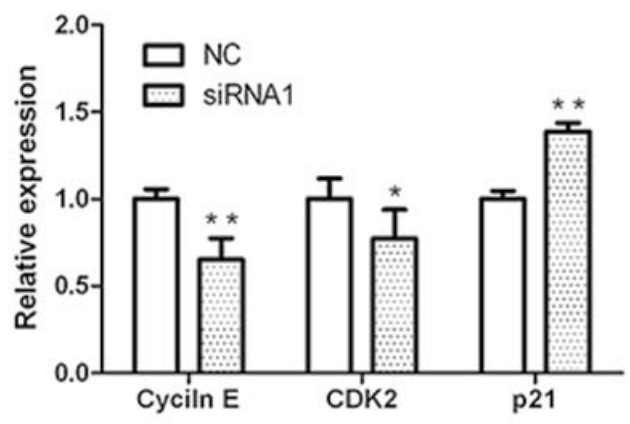

Figure 6. Effects of MCM5 inhibition on cell cycle regulation in SCC-15 cells. (A) Flow cytometry assays were performed to analysis the cell cycle progression when SCC-15 cells transfected with siRNA-MCM5. (B) The bar chart represented the percentage of cells in $\mathrm{G}_{0} / \mathrm{G}_{1}, \mathrm{~S}$, or $\mathrm{G}_{2} / \mathrm{M}_{\mathrm{Phase}}$, as indicated. (C) Expression levels of cell cycle-regulated genes detected by quantitative PCR and normalized to GAPDH. (D) Expression levels of cell cycle-regulated proteins determined using western blotting. $\beta$-actin was used as the loading control. Data are presented as mean $\pm \mathrm{SD}$. ${ }^{*} \mathrm{P}<0.05$, ${ }^{* *} \mathrm{P}<0.01$, vs. control group. MCM5, minichromosome maintenance protein; si, small interfering; NC, negative control; CDK2, cyclin-dependent kinase 2.

To elucidate the mechanism underlying this effect, the expressions levels of cyclin E, cyclin-dependent kinase 2 (CDK2) and p21, related to cell cycle arrest (20), were determined using RT-qPCR. As shown in Fig. 6D, MCM5 inhibition decreased both cyclin $\mathrm{E}$ and $C D K 2 \mathrm{mRNA}$ levels but increased the mRNA expression of p21 significantly. Then, cyclin E and p21 were selected to detect the protein levels using western blotting. As shown in Fig. 6C, cyclin E levels decreased, while p21 levels increased in MCM5-downregulated SCC-15 cells, which was consistent with the RT-qPCR results.

\section{Discussion}

Despite notable progress in cancer research and treatment, the survival rate of patients with OSCC has not significantly improved in the past few decades (2). To date, there are no effective tumor-specific biomarkers for the early detection and prognosis prediction of OSCC (2). Several studies have shown that DEGs serve an important role in the development of tumors in different cancer types (7-9). However, few studies have reported differentially expressed genes in OSCC. The present screened genes that regulate the progression of oral cancer by gene expression profiling and found that 832 genes were dysregulated, of which 416 DEGs were upregulated and 416 were downregulated. DEGs significantly affected 1,490 GO terms and 29 KEGG pathways. MCM5 did not have one of the highest $\log _{2}$ (fold-change) values and $\log _{10}$ (qValues); however, MCM5 is one of the differentially expressed genes in cell cycle signaling pathway, which was the most significant enrichment of differentially expressed genes. Therefore, MCM5, which regulates the cell cycle (10), was selected for further investigation. However, previously published studies on biomarkers in OSCC mainly focused on pathological studies $(2,3,17)$. The present study not only verified the overexpression of MCM5 in OSCC, but also confirmed, using cell experiments, that MCM5 affects cell proliferation by regulating the cell cycle.

MCM5 is a member of the MCM family of proteins and is a component of the starting complex for DNA synthesis (21). MCM5 has been identified as a cell cycle biomarker of aberrant proliferation, which is associated with the progression of various cancer types $(22,23)$. Previously, MCM5 has been found to be overexpressed in numerous human malignancies, such as esophageal (21), thyroid (24) and ovarian cancer (25). For example, increased MCM5 levels in urine sediment cells predicts the presence of bladder cancer $(26,27)$. Inhibition of transcription factor SOX-10 can inhibit the proliferation of skin melanocytes, and MCM5 expression is significantly decreased following downregulation of SOX-10 (11). Moreover, high expression of MCM5 is associated with poor prognosis and poor malignant status in patients with cervical adenocarcinoma $(14,28)$.

It is well known that immunohistochemistry and western blotting are necessary methods to evaluate protein expression (29). 
However, due to a limited number of tissue samples, the present study did not have enough samples for simultaneous qPCR, western blotting and immunohistochemistry detection. Therefore, this is a limitation of the present study. However, the study did perform qPCR to evaluate the expression of MCM5 at the mRNA level. The results demonstrated that $80.0 \%$ of patients with OSCC have high MCM5 expression, which is consistent with the results of the aforementioned studies, indicating that high MCM5 expression may play an important role in the pathogenesis of OSCC. In addition, other members of the MCM family of proteins have also served as biological markers of dysplasia and malignancy, such as glioma, cervical, colorectal, breast, prostate and lung cancer (30-35). Therefore, some researchers even suggested that changes in MCM5 expression may be a sign of cell cycle disorders $(36,37)$.

It is worth noting that some researchers reported that the high expression of MCM family members may be closely related to tumorigenesis and prognosis. For example, MCM2, MCM4, and MCM6 are overexpressed in breast cancer of high histological grades (33). MCM7 expression is a potent prognostic marker in non-small cell lung cancer (38), while MCM5 may be an independent adverse prognostic marker in lung squamous cell carcinoma (34). It is well known that the cell cycle is related to cell proliferation signaling pathways (10). In most tumors, an increase in the expression level of genes encoding proteins that regulate cell proliferation is observed. The abnormal expression of cell-cycle-related genes is associated with infinite proliferation of tumors and poor prognosis $(10,11,20)$. Thus far, only Yu et al (17) reported the relationship between MCM5 and OSCC. The study reported that overexpression of MCM5 in patients with OSCC was significantly associated with tumor site, tumor size, positive lymph node metastasis, later clinical stage, higher histological grade, deeper infiltration depth and peripheral nerve infiltration. However, in the present study, association between high expression of MCM5 with survival, metastasis and poor histologic differentiation was not observed. A Kaplan-Meier analysis of the overall survival rate was not significantly changed in patients with high MCM5 expression compared with patients with low MCM5 expression. It was speculated that due to the small number of samples that there were large differences between individuals. Therefore, in future research, a larger sample size should be used to clarify the relationship between high expression of MCM5 and prognosis of OSCC.

Little is known about the role and potential function of MCM5 in OSCC. In the present study, a loss-of-function analysis was conducted and it was demonstrated that MCM5 participated in regulating cell cycle and cell proliferation in OSCC cells. In fact, inhibiting the expression of MCM5 in SCC-15 cells resulted in the downregulation of cyclin $\mathrm{E}$ and CDK expression and upregulation of p21 expression, which ultimately led to $\mathrm{G}_{2} / \mathrm{M}$ phase arrest in oral cancer cells. These results further verified that MCM5 is highly expressed in patients with OSCC, which promotes the proliferation of OSCC cells and regulates cell cycle. In addition, it was observed that MCM5 was not only expressed in SCC-15 cells, but also expressed in CAL-27 cells (Fig. 4A). Notably, according to the ATCC, the MCM5 gene had no mutations in either of the two cell lines, indicating that the two cell lines selected in this study have similar genetic backgrounds, and could be used for the study of MCM5 cell functions.

Considering MCM5-knockdown experiments in SCC-15 cells with high MCM5 expression received more significant results, SCC-15 cells were selected for follow-up studies. However, analyzing the functional effects of MCM5-knockdown in CAL27 cell lines may provide more information. In addition, both SCC-15 and CAL-27 cells are transformed cell lines. In future investigations, untransformed cell lines for multiple comparisons should be used to clarify the role of MCM5 in OSCC.

Surgical resection is currently the main method to treat OSCC. However, considering the particularity of the oral structure, surgical resection will lead to a huge impact on patients' quality of life $(2,3)$. Therefore, it is important to find effective diagnostic biomarkers for early detection or to develop targeted drugs for OSCC. In the present study, it was reported that MCM5 is overexpressed in OSCC and that MCM5 can affect cell proliferation by regulating cell cycle. Therefore, the results suggested that MCM5 might be one of the important pathogenic factors of OSCC and is expected to be used as a potential tumor marker for OSCC target drugs. The specific mechanism of action of MCM5 is still worthy of further investigation.

Overall, the present study evaluated 832 differentially expressed genes using sequencing patterns in OSCC tumor tissues, and further validated MCM5 upregulated expression in OSCC tissues. By knocking down MCM5 expression in SCC-15 cells, it was revealed that cell proliferation and colony formation was significantly inhibited by inducing $\mathrm{G}_{2} / \mathrm{M}$ phase arrest. The results also suggested that during this process, cyclin E and cell cycle-related gene expression levels were decreased, while p21 was significantly upregulated. Therefore, MCM5 may modulate OSCC cell proliferation by regulating the cell cycle. MCM5 is an important pathogenic factor and might have important role as a potential diagnostic marker or drug target for OSCC.

\section{Acknowledgements}

Not applicable.

\section{Funding}

This study was funded by the National Natural Science Foundation of China (grant no. 81903881), the Bethune Project of Jilin University of China (grant no. 2018B02), the Education Department of Jilin Province (grant no. JJKH20190074KJ), and Department of Science and Technology of Jilin Province (grant no. 20190103086JH and 20200201398JC).

\section{Availability of data and materials}

The datasets used and/or analyzed during the present study are available from the corresponding author on reasonable request. The other datasets generated and/or analyzed during the current study are available in The Cancer Genome Atlas (https://www.cancer.gov/), Gene Ontology (http://geneontology.org/), UniProt (https://sparql.uniprot.org/), NCBI (https://www.ncbi.nlm.nih.gov/) and Kyoto Encyclopedia of Genes and Genomes (https://www.kegg.jp/) databases. 


\section{Authors' contributions}

$\mathrm{HW}, \mathrm{CZ}$ and CL performed the experiments. $\mathrm{HW}, \mathrm{MH}$ and $\mathrm{XW}$ analyzed the data. $\mathrm{MH}$ and $\mathrm{HW}$ wrote the manuscript. $\mathrm{MH}$ and XW designed and supervised the study.

\section{Ethics approval and consent to participate}

The study was approved by The Ethics Committee of China-Japan Union Hospital of Jilin University (Changchun, China). Written informed consent was obtained from all patients who participated in this study.

\section{Patient consent for publication}

Not applicable.

\section{Competing interests}

The authors declare that they have no competing interests.

\section{References}

1. Ruicci KM, Meens J, Sun RX, Rizzo G, Pinto N, Yoo J, Fung K, MacNeil D, Mymryk JS, Barrett JW, et al: A controlled trial of HNSCC patient-derived xenografts reveals broad efficacy of $\mathrm{PI} 3 \mathrm{~K} \alpha$ inhibition in controlling tumor growth. Int J Cancer 145: 2100-2106, 2019.

2. Khurshid Z, Zafar MS, Khan RS, Najeeb S, Slowey PD and Rehman IU: Role of salivary biomarkers in oral cancer detection. Adv Clin Chem 86: 23-70, 2018.

3. Thomson PJ: Perspectives on oral squamous cell carcinoma prevention-proliferation, position, progression and prediction. J Oral Pathol Med 47: 803-807, 2018.

4. Sim F, Leidner R and Bell RB: Immunotherapy for Head and Neck cancer. Oral Maxillofac Surg Clin North Am 31: 85-100, 2019.

5. Yao Y, Chen X, Lu S, Zhou C, Xu G, Yan Z, Yang J, Yu T, Chen W, Qian Y, et al: Circulating long noncoding rnas as biomarkers for predicting head and neck squamous cell carcinoma. Cell Physiol Biochem 50: 1429-1440.

6. Long T, Liu Z, Zhou X, Yu S, Tian H and Bao Y: Identification of differentially expressed genes and enriched pathways in lung cancer using bioinformatics analysis. Mol Med Rep 19: 2020-2040, 2019.

7. Lei B, Zhang XY, Zhou JP, Mu GN, Li YW, Zhang YX and Pang D: Transcriptome sequencing of HER2-positive breast cancer stem cells identifies potential prognostic marker. Tumour Biol 37: 14757-14764, 2016.

8. Zhu L, Guo Q, Jin S, Feng H, Zhuang H, Liu C, Tan M, Liu J, Li X and Lin B: Analysis of the gene expression profile in response to human epididymis protein 4 in epithelial ovarian cancer cells. Oncol Rep 36: 1592-1604, 2016.

9. Xie C, Xu M, Lu D, Zhang W, Wang L, Wang H, Li J, Ren F and Wang C: Candidate genes and microRNAs for glioma pathogenesis and prognosis based on gene expression profiles. Mol Med Rep 18: 2715-2723, 2018.

10. Chen Y, Hennessy KM, Botstein D and Tye BK: CDC46/MCM5, a yeast protein whose subcellular localization is cell cycle-regulated, is involved in DNA replication at autonomously replicating sequences. Proc Natl Acad Sci USA 89: 10459-10463, 1992.

11. Su Z, Zheng X, Zhang X, Wang Y, Zhu S, Lu F, Qu J and Hou L: Sox10 regulates skin melanocyte proliferation by activating the DNA replication licensing factor MCM5. J Dermatol Sci 85: 216-225, 2017.

12. Zhong H, Chen B, Neves H, Xing J, Ye Y, Lin Y, Zhuang G, Zhang SD, Huang J and Kwok HF: Expression of minichromosome maintenance genes in renal cell carcinoma. Cancer Manag Res 9: 637-647, 2017.

13. Liao X, Han C, Wang X, Huang K, Yu T, Yang C, Huang R, Liu Z, Han Q and Peng T: Prognostic value of minichromosome maintenance mRNA expression in early-stage pancreatic ductal adenocarcinoma patients after pancreaticoduodenectomy. Cancer Manag Res 10: 3255-3271, 2018.
14. Wang D, Li Q, Li Y and Wang H: The role of MCM5 expression in cervical cancer: Correlation with progression and prognosis. Biomed Pharmacother 98: 165-172, 2018.

15. Liu H, Takeuchi S, Moroi Y, Lin N, Urabe K, Kokuba H, Imafuku S, Dainichi T, Uchi H, Furue $M$ and Tu Y: Expression of minichromosome maintenance 5 protein in proliferative and malignant skin diseases. Int J Dermatol 46: 1171-1176, 2007.

16. Liao X, Liu X, Yang C, Wang X, Yu T, Han C, Huang K, Zhu G, $\mathrm{Su} \mathrm{H}$, Qin W, et al: Distinct diagnostic and prognostic values of minichromosome maintenance gene expression in patients with hepatocellular carcinoma. J Cancer 9: 2357-2373, 2018.

17. Yu SY, Wang YP, Chang JY, Shen WR, Chen HM and Chiang CP Increased expression of MCM5 is significantly associated with aggressive progression and poor prognosis of oral squamous cell carcinoma. J Oral Pathol Med 43: 344-349, 2014.

18. Livak KJ and Schmittgen TD: Analysis of relative gene expression data using real-time quantitative PCR and the 2(-Delta Delta C(T)) method. Methods 25: 402-408, 2001.

19. Jia CY, Li HH, Zhu XC, Dong YW, Fu D, Zhao QL, Wu W and Wu XZ: MiR-223 suppresses cell proliferation by targeting IGF-1R. PLoS One 6: e27008, 2011.

20. Saleem M, Asif J, Asif M and Saleem U: Amygdalin from apricot kernels induces apoptosis and causes cell cycle arrest in cancer cells: An updated review. Anticancer Agents Med Chem 18: 1650-1655, 2018.

21. Seo YS and Kang YH: The Human replicative helicase, the CMG Complex, as a target for anti-cancer therapy. Front Mol Biosci 5: 26, 2018.

22. Everson M, Magee C, Alzoubaidi D, Brogden S, Graham D, Lovat LB, Novelli M and Haidry R: Minichromosomal maintenance component complex 5 (MCM5) as a marker of barrett's esophagus-related neoplasia: A feasibility study. Dig Dis Sci 64: 2815-2822, 2019.

23. Margalit DN and Lin A: Two sides of the same coin: Head and neck cancer treatment De-Intensification and intensification with induction chemotherapy. Int J Radiat Oncol Biol Phys 102: 1-4, 2018.

24. Mio C, Lavarone E, Conzatti K, Baldan F, Toffoletto B, Puppin C, Filetti S, Durante C, Russo D, Orlacchio A, et al: MCM5 as a target of BET inhibitors in thyroid cancer cells. Endocr Relat Cancer 23: 335-347, 2016.

25. Gakiopoulou H, Korkolopoulou P, Levidou G, Thymara I, Saetta A, Piperi C, Givalos N, Vassilopoulos I, Ventouri K, Tsenga A, et al: Minichromosome maintenance proteins 2 and 5 in non-benign epithelial ovarian tumours: Relationship with cell cycle regulators and prognostic implications. Br J Cancer 97: 1124-1134, 2007.

26. Kelly JD, Dudderidge TJ, Wollenschlaeger A, Okoturo O, Burling K, Tulloch F, Halsall I, Prevost T, Prevost AT, Vasconcelos JC, et al: Bladder cancer diagnosis and identification of clinically significant disease by combined urinary detection of $\mathrm{Mcm} 5$ and nuclear matrix protein 22. PLoS One 7: e40305, 2012.

27. Chen X, Scapa JE, Liu DX and Godbey WT: Cancer-specific promoters for expression-targeted gene therapy: Ran, brms1 and mcm5. J Gene Med 18: 89-101, 2016.

28. Wang D, Wang H, Li Y and Li Q: MiR-362-3p functions as a tumor suppressor through targeting MCM5 in cervical adenocarcinoma. Biosci Rep 38: BSR20180668, 2018.

29. Janardhan K, Jensen $H$, Clayton NP and Herbert RA: Immunohistochemistry in investigative and toxicologic pathology. Toxicol Pathol 46: 488-510, 2018.

30. Hua C, Zhao G, Li Y and Bie L: Minichromosome maintenance (MCM) Family as potential diagnostic and prognostic tumor markers for human gliomas. BMC Cancer 14: 526, 2014.

31. Majid S, Dar AA, Saini S, Chen Y, Shahryari V, Liu J,Zaman MS, Hirata H, Yamamura S, Ueno K, et al: Regulation of minichromosome maintenance gene family by microRNA-1296 and genistein in prostate cancer. Cancer Res 70: 2809-2818, 2010.

32. Das M, Prasad SB, Yadav SS, Govardhan HB, Pandey LK, Singh S, Pradhan S and Narayan G: Over expression of minichromosome maintenance genes is clinically correlated to cervical carcinogenesis. PLoS One 8: e69607, 2013.

33. Issac MSM, Yousef E, Tahir MR and Gaboury LA: MCM2, MCM4, and MCM6 in breast cancer: Clinical utility in diagnosis and prognosis. Neoplasia 21: 1015-1035, 2019.

34. Giaginis C, Georgiadou M, Dimakopoulou K, Tsourouflis G, Gatzidou E, Kouraklis G and Theocharis S: Clinical significance of MCM-2 and MCM-5 expression in colon cancer: Association with clinicopathological parameters and tumor proliferative capacity. Dig Dis Sci 54: 282-291, 2009. 
35. Liu YZ, Wang BS, Jiang YY, Cao J, Hao JJ, Zhang Y, Xu X, Cai Y and Wang MR: MCMs expression in lung cancer: Implication of prognostic significance. J Cancer 8: 3641-3647, 2017.

36. Korkolopoulou P, Givalos N, Saetta A, Goudopoulou A, Gakiopoulou H, Thymara I, Thomas-Tsagli E and Patsouris E: Minichromosome maintenance proteins 2 and 5 expression in muscle-invasive urothelial cancer: A multivariate survival study including proliferation markers and cell cycle regulators. Hum Pathol 36: 899-907, 2005

37. Passerini V, Ozeri-Galai E, de Pagter MS, Donnelly N, Schmalbrock S, Kloosterman WP, Kerem B and Storchová Z: The presence of extra chromosomes leads to genomic instability. Nat Commun 7: 10754, 2016.
38. Aparicio T, Guillou E, Coloma J, Montoya G and Méndez J: The human GINS complex associates with Cdc45 and MCM and is essential for DNA replication. Nucleic Acids Res 37: 2087-2095, 2009.

\section{(†) $\ominus$}

This work is licensed under a Creative Commons Attribution-NonCommercial-NoDerivatives 4.0 International (CC BY-NC-ND 4.0) License. 